\title{
ATP binding cassette transporter retina genotypes and age related macular degeneration: an analysis on exudative non-familial Japanese patients
}

\author{
Sachiko Kuroiwa, Hidenobu Kojima, Takanobu Kikuchi, Nagahisa Yoshimura
}

\begin{abstract}
Aim-To determine whether mutations in the Stargardt's disease gene, ATP binding cassette transporter retina (ABCR) affect the occurrence of age related macular degeneration (AMD) in Japanese nonfamilial patients.

Methods-80 unrelated Japanese patients with AMD (67 males and 13 females; mean age, 67.2 years) diagnosed by indocyanine green angiography and 100 age matched control subjects were studied. Among the AMD patients, $70(87.5 \%)$ had choroidal neovascularisation of exudative type. Genomic DNA was purified from the total blood and 10 exons (exons 11, 23, 29, 32, 34, $37,41,43,44$, and 49) that have been reported to contain AMD associated mutations were amplified by polymerase chain reaction (PCR). The amplicons were analysed by the single strand conformation polymorphism (SSCP) method. The nucleotide sequencing of the amplicons was determined when necessary.

Results-Of the 10 exons, aberrant patterns of SSCP were detected in three exons-exons 29,41 , and 43 . In exon 29 , an aberrant pattern was found in seven of 80 patients $(8.8 \%)$ and eight of 100 controls $(8 \%)$. Sequencing of the PCR products revealed a heterozygous T1428M mutation which has been previously reported as one of the AMD associated mutations. Statistical analysis showed that there was no significant difference in the occurrence of this mutation between these AMD patients and the control groups $(p=0.86)$. In exons 23,41 , and 43 , polymorphisms and sequence variations were found.

Conclusion-No data to support the association between the ABCR gene mutations and $A M D$ of Japanese patients, especially that of the exudative type, were obtained in this molecular genetic analysis.

(Br f Ophthalmol 1999;83:613-615)
\end{abstract}

Ophthalmol of

Shinshu University

School of Medicine,

Matsumoto 390-8621,

Japan

S Kuroiwa

H Kojima

T Kikuchi

N Yoshimura

Correspondence to:

Dr Yoshimura.

Accepted for publication 25 November 1998 atrophy, whereas exudative AMD, characterised by retinal pigment epithelial detachment or choroidal neovascularisation, is less com- mon but is associated with a greater likelihood of severe visual loss. ${ }^{1}$ However, there may be some racial or ethnic differences in the prevalence of dry and exudative types of AMD. ${ }^{2}$ As the number of elderly people in our population increases, the associated social and economic consequences of AMD related visual impairment and blindness are bound to increase, unless successful means of prevention and/or treatment can be found.

Recently, Allikmets et al reported an association of mutations in the Stargardt's disease gene, ATP binding cassette transporter retina (ABCR), with AMD. ${ }^{3}$ They proposed a hypothesis that heterozygous carriers of ABCR mutations develop AMD late in life, while homozygotes develop Stargardt's disease earlier in life. ABCR was identified as a photoreceptor specific gene on chromosome $1 \mathrm{p} 21$ that is mutated in Stargardt's disease (STGD1), which is the most common form of hereditary, recessive macular dystrophy. ${ }^{4}$ Allikmets et al showed that as many as $26(16 \%)$ patients in a group of 167 patients with AMD carried mutations in the ABCR gene, while only $0.45 \%$ of age and ethnic group matched control subjects had this mutations. ${ }^{3}$

What is puzzling, however, is that most AMD patients do not have a familial history of AMD, so it is difficult to accept that as many as $16 \%$ of such patients have mutations in the ABCR gene. Here, we report the results of a mutation study of selected exons of ABCR gene in Japanese non-familial AMD patients.

\section{Materials and methods}

PATIENTS AND CONTROLS

Eighty Japanese patients with AMD (67 males and 13 females; mean age 67.2 years) and 100 age matched Japanese controls (54 males and 46 females; mean age 64.8 years) were recruited from the outpatient clinic, department of ophthalmology, Shinshu University School of Medicine. AMD patients were consecutive but patients who declined to have fluorescein angiography and indocyanine green (ICG) angiography were excluded from the study. Consecutive cataract patients were used as controls. None of the AMD or control subjects were related. All subjects were fully informed about the purpose of the study and informed consent was obtained before entry in this study. Both AMD patients and control subjects underwent full ophthalmological examinations, including slit lamp biomicroscopy, funduscopy, and contact lens biomicroscopic examination of the macula. All eyes with AMD 
Table 1 Prevalence of mutations in the ABCR gene

\begin{tabular}{|c|c|c|c|c|}
\hline & \multicolumn{2}{|l|}{ This study } & \multicolumn{2}{|c|}{ Allikmets et al ${ }^{3}$} \\
\hline & $\begin{array}{l}A M D \\
(n=80)\end{array}$ & $\begin{array}{l}\text { Control } \\
(n=100)\end{array}$ & $\begin{array}{l}A M D \\
(n=167)\end{array}$ & $\begin{array}{l}\text { General } \\
\text { population } \\
(n=220)\end{array}$ \\
\hline \multicolumn{5}{|l|}{ Mutation } \\
\hline $\mathrm{E} 471 \mathrm{~K}$ & $0(0 \%)$ & $0(0 \%)$ & $2(1.2 \%)$ & $0(0 \%)$ \\
\hline R1129L & $0(0 \%)$ & $0(0 \%)$ & $1(0.6 \%)$ & $0(0 \%)$ \\
\hline $\mathrm{T} 1428 \mathrm{M}$ & $7(8.8 \%)$ & $8(8.0 \%)$ & $1(0.6 \%)$ & $0(0 \%)$ \\
\hline R1517S & $0(0 \%)$ & $0(0 \%)$ & $1(0.6 \%)$ & $0(0 \%)$ \\
\hline $\mathrm{I} 1562 \mathrm{~T}$ & $0(0 \%)$ & $0(0 \%)$ & $2(1.2 \%)$ & $0(0 \%)$ \\
\hline G1578R & $0(0 \%)$ & $0(0 \%)$ & $1(0.6 \%)$ & $0(0 \%)$ \\
\hline \multirow{2}{*}{\multicolumn{5}{|c|}{$\rightarrow \mathrm{A}$}} \\
\hline & & & & \\
\hline $\mathrm{R} 1898 \mathrm{H}$ & $0(0 \%)$ & $0(0 \%)$ & $1(0.6 \%)$ & $0(0 \%)$ \\
\hline G1961E & $0(0 \%)$ & $0(0 \%)$ & $6(3.6 \%)$ & $0(0 \%)$ \\
\hline L1970F & $0(0 \%)$ & $0(0 \%)$ & $1(0.6 \%)$ & $0(0 \%)$ \\
\hline $6519 \Delta 11 b p$ & $0(0 \%)$ & $0(0 \%)$ & $1(0.6 \%)$ & $0(0 \%)$ \\
\hline $\mathrm{D} 2177 \mathrm{~N}$ & $0(0 \%)$ & $0(0 \%)$ & $7(4.2 \%)$ & $1(0.45 \%)$ \\
\hline $6568 \Delta \mathrm{C}$ & $0(0 \%)$ & $0(0 \%)$ & $1(0.6 \%)$ & $0(0 \%)$ \\
\hline Totals & $7(8.8 \%)$ & $8(8.0 \%)$ & $26(16 \%)$ & $1(0.45 \%)$ \\
\hline
\end{tabular}

exhibited multiple drusen, geographic atrophy, choroidal neovascular membranes, and vascularised pigment epithelial detachments; control subjects did not have any of these signs. Those patients with multiple drusen, geographic atrophy, or both were classified having the dry type of AMD but patients with previous history or any other clinical signs of other macular diseases were not included in the present study; patients with choroidal neovascular membranes, vascularised pigment epithelial detachments, or both were classified as having the exudative type of AMD. ${ }^{1}$ Fluorescein and ICG angiography, using a scanning laser ophthalmoscope (Rodenstock, Germany), were done on all AMD patients. Choroidal neovascularisation was defined as irregular or well delineated focal or plaque-like zones of ICG hyperfluorescence that persisted to or increased in the late phases of the videoangiogram. ${ }^{5}$ Of the 80 AMD patients, 70 had ICG proved choroidal neovascularisation or a vascularised pigment epithelial detachment. Therefore, these patients were considered to have the exudative type of AMD. The other 10 patients, who had multiple drusen, geographic atrophy, or both, were considered to have the dry type of AMD.

\section{Mutation identification}

Peripheral blood samples were collected from all patients and controls, and genomic DNA was purified with the Qiagen QIAamp Blood Kit (Qiagen, USA). To obtain segments for determination of the nucleotide sequence, the coding regions of the exons that were reported to have AMD associated mutations were amplified by polymerase chain reaction (PCR) from $1 \mu \mathrm{g}$ genomic DNA. ${ }^{3}$ Ten pairs of sense/antisense primers previously described, ${ }^{34}$ were used to amplify the fragments encompassing the coding region of exons $11,23,29,32,34,37,41,43,44$, and 49 . Using Taq DNA polymerase (Perkin Elmer, USA) and the Gene Amp PCR System 2400 (Perkin Elmer) PCR was performed under the following conditions: $95^{\circ} \mathrm{C} 8$ minutes; five cycles of annealing at $62^{\circ} \mathrm{C}$ for 20 seconds, and extension at $72^{\circ} \mathrm{C}$ for 30 seconds; 35 cycles of annealing at $60^{\circ} \mathrm{C}$ for 20 seconds and exten- sion at $72^{\circ} \mathrm{C}$ for 30 seconds; $72^{\circ} \mathrm{C}$ for 5 minutes. ${ }^{3}$ PCR products were diluted with distilled water and analysed by the PCR single strand conformation polymorphism (PCRSSCP) method using the ABI Prism 310 Genetic Analyzer (Perkin Elmer), as described elsewhere. ${ }^{6}$ When necessary, direct sequencing of the PCR fragments was done using the ABI Prism dRhodamine Terminator Cycle Sequencing Ready Reaction Kit (Perkin Elmer). ${ }^{7}$

Mutations were recognised by an approximately equal peak intensity of the two fluorescent dyes at the same site. When a mutation was detected, the entire procedure of PCR and sequencing was repeated, so the existence of a mutation was confirmed.

\section{Results}

A total of 10 exons of the ABCR gene were analysed for this mutation study; each of these exons has been reported to have AMD associated mutations. ${ }^{3}$ Of the 10 exons studied, aberrant patterns of SSCP were detected in three: exons 29, 41, and 43. In exon 29, seven of 80 AMD patients and eight of 100 controls were found to have aberrant patterns by SSCP. Of the seven AMD patients, six had the exudative type and one had the dry type. Sequencing of the PCR products revealed a heterozygous $\mathrm{C} \rightarrow \mathrm{T}$ mutations at the second nucleotide position in the codon corresponding to the 1428th amino acid residue of the ABCR protein, resulting in an amino acid change from threonine to methionine (T1428M) (Table 1). This mutation was reported by Allikmets et al to be one of the AMD associated mutations. ${ }^{3}$ Statistical analysis, using the $\chi^{2}$ test, however, showed that there was no statistically significant difference in the occurrence of this mutation between our AMD patients and control groups $(p=0.86$, odds ratio $=1.10)$.

In exons 41 and 43, polymorphisms were found. As shown in Table 2, a nucleotide substitution was found in exon 41. Nucleotide sequencing showed that there were both heterozygous and homozygous $\mathrm{G} \rightarrow \mathrm{C}$ transversion at the third nucleotide position in the codon corresponding to the 1844th amino acid residue; but transversion did not cause the amino acid change (L1844L). Another polymorphism was found in exon 43 . The third nucleotide position in the codon corresponding to the 1948th amino acid residue A was changed to G. Both heterozygous and homozygous transitions were found, but these also were not causative of the amino acid change (P1948P). All of these substitutions were found in both AMD patients and normal controls. Interestingly, patients who had the L1884L transversion always had the P1948P transition as well (Table 2).

In exon 23, three sequence variations were detected by nucleotide sequencing of the PCR products, but not detected by SSCP, were found in all AMD patients as well as normal controls. These variations may be innocent ones found in the Japanese population or may be sequence variations (Table 2). At any rate, no AMD associated alterations were detected 
Table 2 Polymorphisms and sequence variations found in this study

\begin{tabular}{llllll}
\hline Mutations & Location & $\begin{array}{l}\text { Nucleotide } \\
\text { changes }\end{array}$ & Genotype & AMD $(n=80)$ & $\begin{array}{l}\text { Control } \\
(n=100)\end{array}$ \\
\hline P1116S & exon 23 & CAC $\rightarrow$ CTT & homozygote & $80(100 \%)$ & $100(100 \%)$ \\
H1125L & exon 23 & CAC $\rightarrow$ CTC & homozygote & $80(100 \%)$ & $100(100 \%)$ \\
Q1126L & exon 23 & CAA $\rightarrow$ CTT & homozygote & $80(100 \%)$ & $100(100 \%)$ \\
L1844L & exon 41 & CTG $\rightarrow$ CTC & homozygote & $2(2.5 \%)$ & $1(1 \%)$ \\
L1844L & exon 41 & CTG $\rightarrow$ CTC & heterozygote & $17(21.3 \%)$ & $28(28 \%)$ \\
P1948P & exon 43 & CCA $\rightarrow$ CCG & homozygote & $2(2.5 \%)$ & $1(1 \%)$ \\
P1948P & exon 43 & CCA $\rightarrow$ CCG & heterozygote & $17(21.3 \%)$ & $28(28 \%)$ \\
\hline
\end{tabular}

in exons $11,23,29,32,34,37,41,43,44$, or 49 in our patient group.

\section{Discussion}

In this study, we performed a mutation analysis of the ABCR gene of 80 unrelated AMD patients and 100 race and age matched control subjects. Of 51 exons of the ABCR gene, 10 that have been reported to have AMD associated mutations were studied. ${ }^{3}$ The only missense mutation found was a T1428M mutation in exon 29. Allikmets et al reported that this mutation was found in one of 167 AMD patients, and that no such mutation was found in the general population. ${ }^{3}$ However, in our series, seven of $80(8.8 \%)$ AMD patients and eight of $100(8.0 \%)$ of the control group did, in fact, showed this mutation (Table 1), which was not a statistically significant difference. Other alterations found were mostly polymorphisms or sequence variations. These were found in exons $23,41,43$, and did not correspond to the $13 \mathrm{AMD}$ associated mutations reported by Allikmets et al. ${ }^{3}$ Apparently, there is heterogeneity in the ABCR gene. Indeed, all of the AMD and the control subjects in our series showed different nucleotide sequences from that reported for exon $23 .^{4}$

In our series, all of the AMD patients were studied by fluorescein and ICG angiography; these tests were indispensable in making the diagnosis of AMD and with these tests a definite diagnosis of exudative AMD could be made. Even with fluorescein and ICG angiography, however, there is some ambiguity in the diagnosis of dry AMD; old central serous chorioretinopathy could be mistaken for dry AMD and Stargardt's disease in elderly patients could masquerade as dry AMD. ${ }^{8}$ Furthermore, there is no clear distinction between dry AMD and normal aging changes of the macula. Therefore, it is reasonable to focus on exudative AMD, rather than dry type, when we try to find causative genes of AMD. In the present study, 70 of 80 patients had ICG angiography proved changes of choroidal neovascularisation, and thus most of our patients are of the exudative rather than the dry type. Thus, as Allikmets et al focused on dry AMD, the subjects of the present study are somewhat different. ${ }^{3}$ Therefore, we cannot deny a possibility that ABCR genotypes are associated with dry AMD but not with exudative AMD.

ABCR is identical to a previously identified rod outer segment protein called rim protein $(\mathrm{RmP}),{ }^{9-12}$ which is known to colocalise with
peripherin/RDS. ${ }^{13-15}$ Mutations in proteins localised in the visual cells, especially within rod outer segment, have been shown to be associated with retinitis pigmentosa. Examples include rhodopsin and peripherin/RDS. ${ }^{13-15}$ Because ABCR is localised in the rod outer segments, the gene is a good candidate to be the causative gene of retinitis pigmentosa. Very recently, Martínez-Mir et al ${ }^{16}$ reported patients in whom a homozygous mutation in exon 13 of ABCR was identified, and Cremers et al reported splice site mutations in the ABCR gene in autosomal recessive retinitis pigmentosa and cone-rod dystrophy. ${ }^{17}$ Although our present study does not exclude the possibility that mutations in other exons of ABCR are associated with dry or even exudative AMD, studies on the association of ABCR mutations with retinitis pigmentosa and other forms of chorioretinal atrophy, together with further studies on AMD, would certainly increase our understanding of the role of ABCR in these and other retinal disorders.

1 Bird AC, Bressler NM, Bressler SB, et al. An international classification and grading system for age-related maculopathy and age-related macular degeneration. Surv Ophthalmol 1992;39:367-74.

2 Bird AC. Prevalence of AMD in different racial groups. In: Pathogenesis and treatment of age-related macular degeneration: current knowledge and leads for future research. Baltimore: Johns Hopkin University, 1998; Section 2.

3 Allikmets R, Shroyer NF, Singh N, et al. Mutation of the Stargardt disease gene (ABCR) in age-related macular degeneration. Science 1997;277:1805-7.

4 Allikmets R, Singh N, Sun H, et al. A photoreceptor cell-specific ATP-binding transporter gene (ABCR) is mutated in recessive Stargardt macular dystrophy. Nature Genet 1997;15:236-46.

5 Guyer DR, Yannuzzi LA, Slakter JS, et al. Classification of choroidal neovascularization by digital indocyanine green videoangiography. Ophthalmology 1996;103:2054-60.

6 Inazuka M, Wenz HM, Sakabe $M$, et al. A streamlined mutation detection system: multicolor post-PCR fluorescence labeling and single-strand conformational polymorphism analysis by capillary electrophoresis. Genome Res 1997; 7:1094-103.

7 Ahmad W, Haque MF, Brancolini V, et al. Alopecia universalis associated with a mutation in the human hairless gene. Science 1998;279:720-4.

8 Dryja TP, Briggs CE, Berson EL, et al. Technical comment summaries. ABCR gene and age-related macular degeneration. Science 1998;279:1107.

9 Papermaster DS, Converse CA, Zorn CM. Biosynthetic and immunochemical characterization of a large protein in frog and cattle rod outer segment membranes. Exp Eye Res 1976;23:105-15.

10 Illing M, Molday LL, Molday RS. The $220-\mathrm{kDa}$ rim protein of retinal rod outer segments is a member of the ABC transporter superfamily. F Biol Chem 1997;272:10303-10.

11 Azarian SM, Travis GH. The photoreceptor rim protein is an ABC transporter encoded by the gene for recessive

12 Thomson JL, Brzeski H, Dunbar B, et al. Photoreceptor rim protein: partial sequences of cDNA show a high degree of similarity to ABC transporters. Curr Eye Res 1997;16:7415.

13 Dryja TP, McGee TL, Reichel E, et al. A point mutation of the rhodopsin gene in one form of retinitis pigmentosa. Nature 1990;343:364-6.

14 Kajiwara K, Berson EL, Dryja TP. Digenic retinitis pigmentosa due to mutation at the unlinked peripherin/RDS and ROM1 loci. Science 1994;264:1604-8.

15 Dryja TP, Hahn LB, Kajiwara K, et al. Dominant and digenic mutations in the peripherin/RDS and ROM1 genes in retinitis pigmentosa. Invest Ophthalmol Vis Sci 1997;38: 1972-82.

16 Martínez-Mir A, Paloma E, Allikmets R, et al. Retinitis pigmentosa caused by a homozygous mutation in the Stargardt disease gene ABCR. Nature Genet 1998;18:11-2.

17 Cremers FPM, van de Pol, DJR, van Driel $M$, et al. Autosomal recessive retinitis pigmentosa and cone-rod dystrophy caused by splice site mutations in the Stargardt's disease gene ABCR. Hum Mol Genet 1998;7:355-62. 